\title{
Prognosis of Incidental Brain Metastases in Patients With Advanced Renal Cell Carcinoma
}

Ritesh R. Kotecha, MD'; Ronan Flippot, MD, MSc²; Taylor Nortman, $\mathrm{BS}^{1}$; Annalisa Guida, MD²,3 Sujata Patil, PhD4; Bernard Escudier, MD²; Robert J. Motzer, MD¹; Laurence Albiges, MD²; and Martin H. Voss, MD

\section{ABSTRACT}

Background: Metastatic renal cell carcinoma (mRCC) management guidelines recommend brain imaging if clinically indicated and the rate of occult central nervous system (CNS) metastasis is not welldefined. Early detection could have major therapeutic implications, because timely interventions may limit morbidity and mortality. Patients and Methods: A retrospective review was performed to characterize patients with $\mathrm{mRCC}$ incidentally diagnosed with asymptomatic brain metastases during screening for clinical trial participation at Gustave Roussy and Memorial Sloan Kettering Cancer Center. Descriptive statistics and time-to-event methods were used to evaluate the cohort. Results: Across 68 clinical trials conducted between 2001 and 2019 with a median 14.1-month follow-up, 72 of 1,689 patients (4.3\%) with mRCC harbored occult brain metastases. The International Metastatic RCC Database Consortium (IMDC) risk status was favorable (26\%), intermediate (61\%), and poor (13\%), and $86 \%$ of patients had $\geq 2$ extracranial sites of disease, including lung metastases in $92 \%$ of patients. CNS involvement was multifocal in $38.5 \%$ of patients, and the largest brain metastasis was $>1 \mathrm{~cm}$ in diameter in $40 \%$ of the cohort. Localized brain-directed therapy was pursued in $93 \%$ of patients, predominantly radiotherapy. Median overall survival was 10.3 months (range, 7.0-17.9 months), and the 1-year overall survival probability was $48 \%(95 \% \mathrm{Cl}, 37 \%-62 \%)$. IMDC risk and number or size of lesions did not correlate with survival (log-rank, $P=.3, P=.25$, and $P=.067$, respectively). Conclusions: This large multi-institutional mRCC cohort study identified occult brain metastasis in a notable proportion of patients $(4.3 \%)$ and highlights that the risk of asymptomatic CNS involvement extends to those with favorable risk features per IMDC risk assessment. These data provide rationale for brain screening in patients with advanced RCC.

J Natl Compr Canc Netw 2021;19(4):432-438 doi: $10.6004 /$ jnccn.2020.7634

\footnotetext{
${ }^{1}$ Department of Medicine, Memorial Sloan Kettering Cancer Center, New York, New York; ${ }^{2}$ Department of Cancer Medicine, Gustave Roussy, Paris-Saclay University, Villejuif, France; ${ }^{3}$ Department of Oncology and Hematology, University of Modena and Reggio Emilia, Modena, Italy; and ${ }^{4}$ Department of Epidemiology and Biostatistics, Memorial Sloan Kettering Cancer Center, New York, New York.
}

\section{Background}

Brain metastases are a critical site of disease progression in patients with metastatic renal cell carcinoma (mRCC). Large institutional and population-based studies estimate the incidence of brain metastasis to be $5 \%$ to $20 \%,{ }^{1-6}$ and variability in incidence rates partly relates to the fact that brain metastases are typically diagnosed when new symptoms prompt evaluation. Without standardized screening measures, these historical rates provide limited guidance to clinicians, because the true incidence may not include patients with asymptomatic disease who do not undergo evaluation. Efforts to comprehensively identify this patient population are needed, given that multidisciplinary clinical management is often prioritized to limit morbidity and mortality associated with local progression.

Per national and international guidelines standardizing mRCC management, ${ }^{7-10}$ brain imaging is recommended at the clinician's discretion and hence is often not performed in the absence of symptoms. However, brain imaging is commonly mandated as a part of eligibility assessments for clinical trial entry. Such uniform screening provides a systematic opportunity to investigate the rate of incidental brain metastasis in mRCC and characterize outcomes in this specific patient population.

\section{Patients and Methods}

After obtaining Institutional Review Board approval at Gustave Roussy and Memorial Sloan Kettering Cancer Center, a retrospective multicenter chart review was performed at the participating centers. A combined deidentified database was constructed of patients with $m R C C$ who required brain imaging as a part of eligibility screening for participation in institutional clinical trials and were incidentally diagnosed with brain metastases. Clinical trials that did not require brain imaging and patients who were deemed ineligible for trial participation for reasons other than brain metastasis were excluded. Chart review was performed to confirm the absence of neurologic symptoms at the time of the 
screening, including numbness, weakness, dizziness, balance problems, headache, or altered mental status. Documented physical examinations were also screened for any physical signs related to these symptoms. Baseline clinical features collected included demographics and International Metastatic RCC Database Consortium (IMDC) risk status at study screening. Brain metastases were characterized by number, size of largest metastasis (per longest axis diameter), and presence or absence of associated vasogenic edema through review of institutional radiology reports. Patients were managed according to best practices at each center, and site-specific therapeutic interventions and outcomes were recorded. Data cutoff was November 1, 2019.

Continuous variables were summarized using median with minimum and maximum, and categorical data were tabulated using frequencies and percentages. Median overall survival (OS) and 95\% confidence intervals were estimated using the Kaplan-Meier method. Survival was measured from diagnosis of brain metastasis until death from any cause, and patients were censored at the time of last follow-up if alive or without subsequent follow-up data. Log-rank testing was used to compare survival outcomes by clinical variables. $\mathrm{R}$ version 3.6.1 (R Foundation for Statistical Computing) was used for statistical analyses.

\section{Results}

\section{Patient Characteristics}

From 2001 through 2019, 68 clinical trials conducted at Gustave Roussy and Memorial Sloan Kettering Cancer Center for patients with mRCC included mandatory brain screening by CT/MRI at study entry. Among 1,689 patients screened, 72 (4.3\%; 95\% CI, 3.3\%-5.3\%) were found to have incidental brain metastases without documented neurologic symptoms upon dedicated chart review. Of these 72 patients, $54(75 \%)$ were male, and the median age at mRCC diagnosis was 56 years (range, 37-77 years) (Table 1). At the time of trial screening, 16 patients (26\%) had IMDC favorable-risk disease, 37 (61\%) had intermediate-risk disease, and $8(13 \%)$ had poor-risk disease. Data were incomplete for full IMDC risk calculation in 11 patients. A total of 63 patients (88\%) had undergone nephrectomy, and all patients included in this analysis had a clear-cell histologic subtype, with $6(8 \%)$ harboring sarcomatoid dedifferentiation. At the time of initial RCC diagnosis, 43 patients $(60 \%)$ presented with metastatic disease, and at the time of brain metastasis diagnosis, 62 (86\%) had more than one site of extracranial disease on radiographic assessment. The most common extracranial disease site was lung in 66 patients $(92 \%$ of those with incidental brain metastases), followed by liver and bone (18 patients each [25\%]). Brain metastases were diagnosed in 22 of

\section{Table 1. Baseline Patient Characteristics}

\begin{tabular}{|c|c|}
\hline Characteristic & n (\%) \\
\hline Patients, N & 72 \\
\hline Median age at mRCC diagnosis (range), y & $56(37-77)$ \\
\hline $\begin{array}{l}\text { Median time from } \mathrm{mRCC} \text { diagnosis to } \mathrm{BM} \\
\text { diagnosis (range), mo }\end{array}$ & $16.1(0.2-162)$ \\
\hline \multicolumn{2}{|l|}{ Available IMDC risk at study screening } \\
\hline Favorable & $16(26)$ \\
\hline Intermediate & $37(61)$ \\
\hline Poor & $8(13)$ \\
\hline Unknown & 11 \\
\hline History of prior nephrectomy & $63(88)$ \\
\hline \multicolumn{2}{|l|}{ Systemic therapy } \\
\hline Treatment-naïve & $23(32)$ \\
\hline 1 prior systemic treatment & $31(43)$ \\
\hline$\geq 2$ prior systemic treatments & $18(25)$ \\
\hline Clear-cell histology & $72(100)$ \\
\hline Sarcomatoid features & $6(8)$ \\
\hline \multicolumn{2}{|l|}{ Tumor grade } \\
\hline 1 & $1(2)$ \\
\hline 2 & $13(24)$ \\
\hline 3 & $24(44)$ \\
\hline 4 & $17(31)$ \\
\hline Unknown & 17 \\
\hline Stage IV at initial diagnosis & $43(60)$ \\
\hline \multicolumn{2}{|c|}{ Extracranial organ sites of disease at BM diagnosis } \\
\hline 1 & $10(14)$ \\
\hline 2 & $33(46)$ \\
\hline$\geq 3$ & $29(40)$ \\
\hline Presence of lung metastases & $66(92)$ \\
\hline Presence of liver metastases & $18(25)$ \\
\hline Presence of bone metastases & $18(25)$ \\
\hline
\end{tabular}

Abbreviations: BM, brain metastasis; IMDC, International Metastatic RCC Database Consortium; mRCC, metastatic renal cell carcinoma.

832 patients (2.6\%) screened for first-line studies, and in 50 of 857 patients $(5.8 \%)$ screened in the treatmentrefractory setting.

\section{Brain Metastasis Characteristics}

All patients were confirmed by chart review to be asymptomatic at study screening. A total of 45 patients $(63 \%)$ presented with a solitary lesion, $10(14 \%)$ presented with 2 brain metastases, and 17 (24\%) presented with $\geq 3$ lesions. Associated edema by radiographic review was found in 57 patients (79\%). A total of 67 patients $(93 \%)$ had imaging evaluable for size measurements per expert radiologist, and $40(60 \%)$ of those patients had $\leq 1 \mathrm{~cm}$ disease in the longest-axis measurement of the 
largest lesion. Additional details regarding brain-specific disease are noted in Table 2.

\section{Site-Directed Therapies}

Of the available site-directed therapy follow-up data in 63 patients, 52 (83\%) were administered corticosteroids. Of the 63 patients, 61 had additional follow-up available and 57 (93\%) underwent site-specific therapy, including radiotherapy in 49 patients (86\%), surgical resection in $6(11 \%)$, and combination surgery plus radiotherapy in 2 (4\%). Median time from detection of brain metastasis to start of site-directed therapy was 30 days (range, 4-262 days; Table 3).

\section{Survival Outcomes in Patients With Incidental Brain Metastasis}

A total of 62 patients $(86 \%)$ had died in this cohort at the time of data cutoff, and the median follow-up for the 10 censored patients was 14.1 months (range, $0-75$ months). Median OS was 10.3 months (range, 7.0-17.9 months) and the 1-year OS rate was $48 \%(95 \%$ CI, $37 \%-62 \%$; Figure 1). When patients were stratified by IMDC risk status, the survival data for favorable-risk, intermediaterisk, or poor-risk disease were not significantly different when compared using log-rank testing ( $P=.3$; Figure 2). Median OS for the favorable-risk, intermediate-risk, and poor-risk groups was 12.7 months (range, 4.8 months-not reached), 12.4 months (range, 7.4-19.7 months), and 4.5 months (range, 3.8 months-not reached), respectively. The 1-year OS probability of favorable-risk, intermediate-risk, and poor-risk disease was 53\% (95\% CI, 33\%-86\%), 52\% (95\% CI, 38\%-71\%), and 29\% (95\% CI, 9\%-92\%), respectively.

OS did not significantly differ between patients with solitary or multifocal disease (log-rank, $P=.25$ ), with a 1 -year OS probability of $57 \%$ (95\% CI, 44\%-74\%) and $33 \%$ (95\% CI, 19\%-58\%), respectively (Figure 3A).

\section{Table 2. Brain Metastasis Characteristics}

\begin{tabular}{|lc|}
\hline Characteristic & $\mathbf{n}(\%)$ \\
\hline Patients, N & 72 \\
\hline Solitary lesion & $45(63)$ \\
\hline $\begin{array}{l}\text { Multifocal } \\
2 \text { lesions }\end{array}$ & $27(38)$ \\
\hline$\geq 3$ lesions & $10(14)$ \\
\hline Associated edema present & $17(24)$ \\
\hline $\begin{array}{l}\text { Size of brain metastases (longest axis, largest lesion) } \\
\quad \leq 1 \mathrm{~cm}\end{array}$ & $57(79)$ \\
\hline$>1 \mathrm{~cm}$ & $40(56)$ \\
\hline Unknown & $27(38)$ \\
\hline If $>1 \mathrm{~cm}$, median size of CNS metastasis (range), cm & $1.75(1.1-5.1)$ \\
\hline
\end{tabular}

Abbreviation: CNS, central nervous system.

\section{Table 3. Brain Metastasis Site-Directed Therapy}

\begin{tabular}{|c|c|}
\hline Brain Metastasis Treatment Summary & n (\%) \\
\hline Patients, N & 61 \\
\hline Corticosteroids administered ${ }^{a}$ & $52(83)$ \\
\hline Site-specific therapy administered & $57(93)$ \\
\hline Stereotactic radiotherapy & $41(72)$ \\
\hline Whole-brain radiotherapy & $8(14)$ \\
\hline Surgical resection & $6(11)$ \\
\hline Radiotherapy + surgical resection & $2(4)$ \\
\hline $\begin{array}{l}\text { Median time between imaging and site-directed } \\
\text { therapyb (range), } d\end{array}$ & $30(4-262)$ \\
\hline
\end{tabular}

aMedically directed treatment data were available for 63 patients. bRadiotherapy or surgery.

Median OS for patients with solitary disease and multifocal disease was 14.2 months (range, 9.5-21.6 months) and 5.8 months (range, 4.1-18 months), respectively. When patients were further stratified by degree of multifocal disease (Figure 3B), those with $>2$ brain metastases were found to have a 1-year OS probability of $21 \%(95 \%$ CI, $8 \%-57 \%)$ and a median OS of 4.4 months (range, 3.8 months-not reached). The OS comparison between patients with solitary disease and those with $\geq 2$ brain metastases also did not show any significant difference (log-rank, $P=.067$ ). When stratified by patients with $\leq 1$ or $>1 \mathrm{~cm}$ disease, no differences were seen in OS (logrank, $P=.21$ ) (Figure 4). The 1-year OS probability and median OS for patients with $\leq 1$ versus $>1 \mathrm{~cm}$ disease were $49 \%$ (95\% CI, 35\%-68\%) and 12.7 months (range, 7.2-33.5 months) versus 52\% (95\% CI, 36\%-76\%) and 10.6 months (range, 5.9-17.9 months), respectively. Survival outcomes integrating size and number are summarized in Figure 5; no formal statistical comparison was performed given the limited subgroup size.

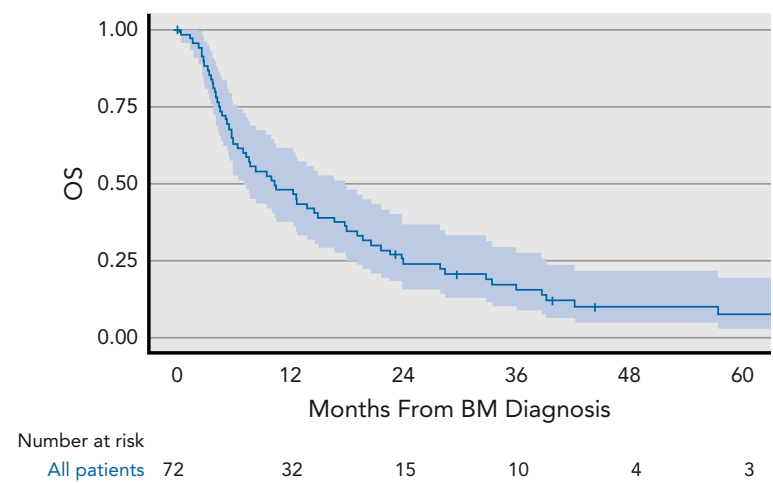

Figure 1. OS from diagnosis of brain metastasis in RCC. OS was assessed using the Kaplan-Meier method, with a median follow-up of 14 months (95\% Cl, 3.7-75.6).

Abbreviations: BM, brain metastasis; OS, overall survival; RCC renal cell carcinoma. 


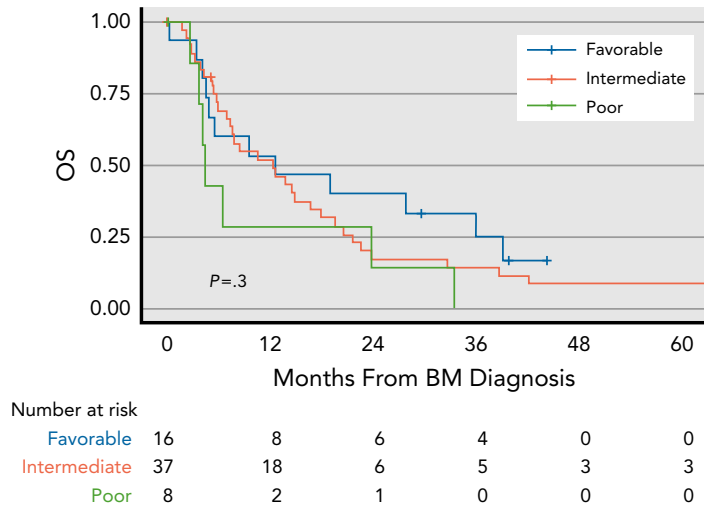

Figure 2. OS by IMDC risk score from diagnosis of brain metastasis. IMDC risk status was assessed at trial screening, and OS was assessed using the Kaplan-Meier method.

Abbreviations: BM, brain metastasis; IMDC, International Metastatic Renal Cell Carcinoma Database Consortium; OS, overall survival.

\section{Discussion}

This large, multi-institutional retrospective study of patients with mRCC who underwent mandatory brain screening as part of clinical trial entry provided a unique opportunity to systematically assess the rate of incidental brain metastatic disease. Among the 1,689 patients who underwent screening, $72(4.3 \%)$ were found to harbor asymptomatic brain metastases, with a higher incidence among patients previously treated with systemic therapy. This cohort of patients had a median 1-year OS probability of $48 \%$ and a median OS of 10.3 months. Application of IMDC risk status and other brain-specific metrics, including metastasis size and number, were not significantly associated with OS outcomes.

These results add to the increasing body of literature evaluating the incidence of brain metastases among

\section{A}

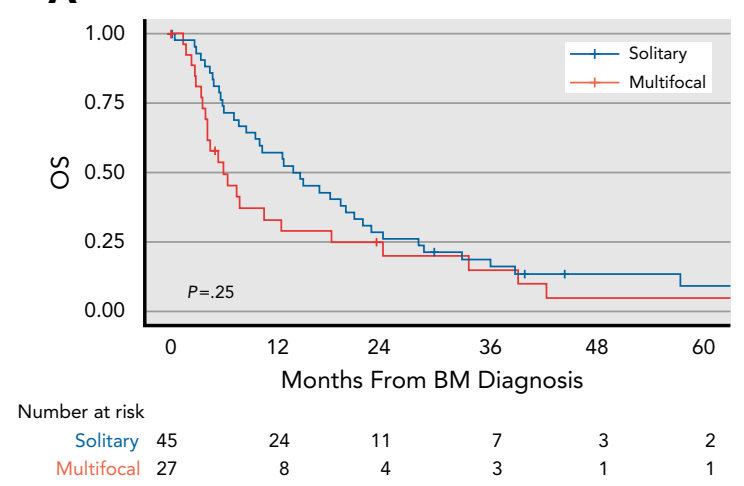

patients with mRCC and their outcomes, and add clarity to a commonly overlooked group of patients: those who present incidentally with central nervous system (CNS) disease. Prior reports exploring the SEER program have estimated that $12.1 \%$ of patients with mRCC had brain metastasis at diagnosis, with a total of $20.4 \%$ in the total mRCC population. ${ }^{2}$ Other patient series have identified relative similar incidences, ${ }^{1,3}$ with a single institutional dataset highlighting that $32.6 \%$ of a total cohort of patients with brain metastasis $(7.4 \%$ of 1,855 patients with mRCC evaluated) had asymptomatic disease presentation. ${ }^{3}$ A more contemporary dataset of patients with mRCC showed an overall frequency of brain metastasis of $28.4 \%$, which may also reflect the adoption of improved imaging techniques for detection, such as MRI, and an increased awareness of the benefit of brain screening. ${ }^{4}$ Interestingly, the single institutional dataset showed that IMDC risk status remained prognostic, particularly in the poor-risk disease group, contrary to the current study's finding. This difference may reflect discrepancies in the patient population, given that the dataset presented here consists of asymptomatic patients only and may differ from an all-comer cohort. In addition, our cohort exclusively included patients considered for clinical trial participation, which may introduce a selection bias toward more fit patients. Notably, in KEYNOTE-426, the registration phase III study of pembrolizumab + axitinib, 22 patients $(2.1 \%$ of the screening population) were not eligible due to active CNS metastases and/or carcinomatous meningitis at trial screening, and this remained the most common reason that patients were unable to participate due to an exclusion criteria. ${ }^{11}$ In the phase III METEOR trial of cabozantinib in the second-line setting, 264 of 922 patients $(28.6 \%)$ were ineligible at screening, and brain metastases requiring treatment were a primary

\section{B}

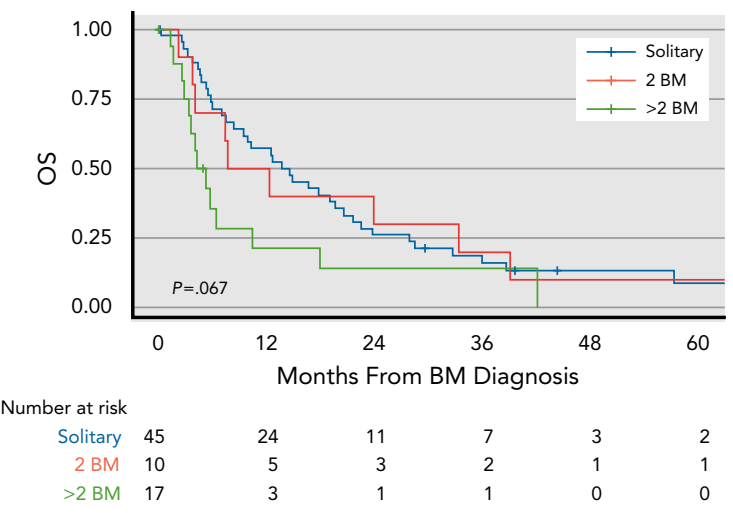

Figure 3. OS by solitary versus multifocal brain metastases. Patients were grouped by either $(A)$ solitary versus multifocal disease or (B) further stratified by the number of specific lesions. Median OS was not significantly different between these groups by log-rank testing ( $P=.25$ and $P=.067$, respectively).

Abbreviations: BM, brain metastasis; OS, overall survival. 


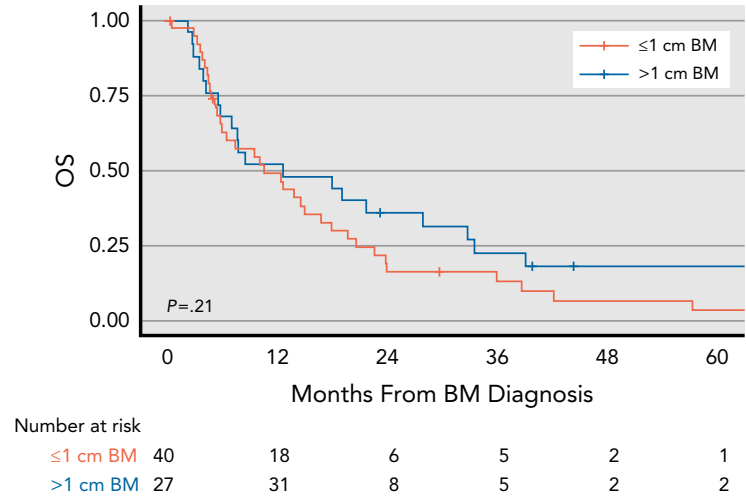

Figure 4. OS by size of largest brain metastasis. Radiographic imaging was reviewed, and the largest isolated brain metastasis in the long axis was used to stratify subcentimeter and $>1 \mathrm{~cm}$ disease. Abbreviations: BM, brain metastasis; OS, overall survival.

reason. ${ }^{12}$ Although clinical information detailing whether those patients had incidental disease is unavailable for both trials, the rate seen in the first-line setting is consistent with our findings ( $2.6 \%$ in our cohort).

Several factors have been proposed to be associated with the presence of brain-specific metastatic disease, including sarcomatoid dedifferentiation, primary tumor size, regional node involvement, and thoracic and osseous sites of extracranial disease. ${ }^{6,13}$ Similar to the latter, we found a notably high proportion of patients with concurrent metastases to the lung (92\%). Furthermore, in our cohort, occult CNS disease developed in the background of stage IV disease at initial diagnosis at a remarkably higher rate than in historical controls $(60 \%$ vs $30 \%$, respectively). ${ }^{14,15}$ Although we were unable to compute the incidence per IMDC risk category (IMDC risk scores were unavailable for all 1,689 patients screened), $26 \%$ of patients in our cohort had IMDC favorable-risk disease at the time of screening and brain metastasis diagnosis. This finding suggests that screening considerations in routine practice should not be limited to patients deemed to have high-risk disease per standard criteria, and should be considered in patients with high metastatic burden.

In terms of brain-specific characteristics of metastatic disease, lesion size has previously been associated with symptoms, ${ }^{3,5}$ whereas lesion number has been associated with lower OS. ${ }^{16}$ In our cohort, we compared features of systemic disease and CNS involvement with OS outcomes. Although numerically, brain-specific features including lesion number and size differed in terms of their association with OS, the presence alone of brain metastases seemed to largely impact outcomes in this asymptomatic disease cohort rather than individualized CNS disease metrics. Although no significant differences were identified among IMDC risk groups, the median OS
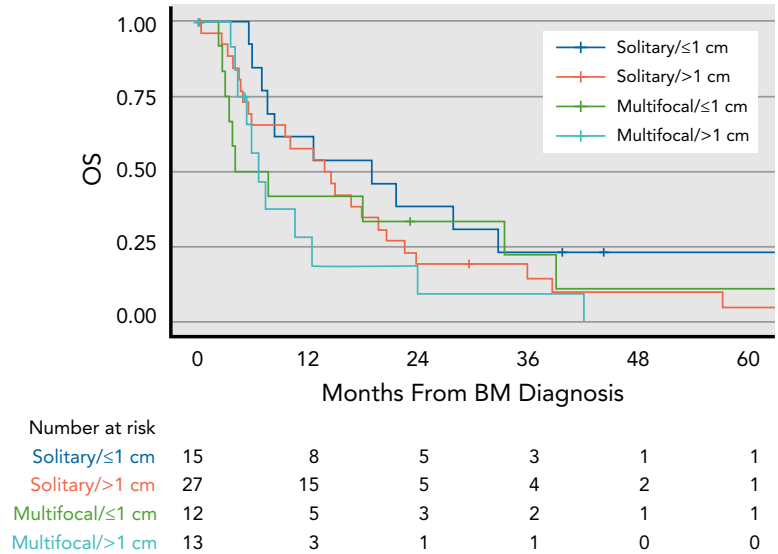

$\begin{array}{rll}8 & 5 & 3 \\ 15 & 5 & 4 \\ 5 & 3 & 2 \\ 3 & 1 & 1\end{array}$

$\begin{array}{ll}1 & 1 \\ 2 & 1 \\ 1 & 1 \\ 0 & 0\end{array}$

Figure 5. OS by size and focality of brain metastases. The patient cohort was subdivided based on solitary or multiple lesions and $\leq 1$ $\mathrm{cm}$ disease. Median OS for the solitary $\leq 1 \mathrm{~cm}$ group and multifocal $>1 \mathrm{~cm}$ group was 19 months (range, 7.7 months-NR) and 6.5 months (range, 5.3 months-NR), respectively. Formal statistical comparison was not performed.

Abbreviations: BM, brain metastasis; NR, not reached; OS, overall survival.

was low even among favorable-risk and intermediaterisk groups. This finding highlights that patients with brain metastasis may have a relatively poor prognosis even when diagnosed in the occult setting, and the presence of metastasis alone for this disease site bears significant weight.

The landscape of therapeutic options for patients with mRCC continues to rapidly evolve, with impressive response rates and superior survival outcomes seen in response to combination immune checkpoint inhibitors $^{17}$ and combination vascular endothelial growth factor receptor tyrosine kinase inhibitor (VEGFR-TKI) plus immune checkpoint inhibitor therapies. ${ }^{12,18}$ However, patients with brain metastases are usually excluded from studies, and therefore experience with and activity of these agents in this setting (either asymptomatic or symptomatic) remain limited. Retrospective data suggest that TKI use within 30 days may increase the rate of radiation necrosis ${ }^{19}$ or could lead to disease flare. ${ }^{20}$ However, anecdotal case reports have also highlighted monotherapy responses using next-generation VEGFRTKIs such as cabozantinib in radioresistant brain tumors. ${ }^{21}$ In a review of prospective studies of patients with brain metastases, sunitinib monotherapy was not shown to have an objective intracranial response. ${ }^{22}$ Updated results have been reported from the NIVOREN brain metastasis cohort, a prospective study assessing nivolumab monotherapy in both patients who were treatment-naïve and those treated for brain metastasis. ${ }^{23}$ In that cohort of 73 patients, the intracranial response rate was $12 \%$ for patients who were treatment-naïve, with no response seen in tumors $>1 \mathrm{~cm}$; the median intracranial progression-free survival (PFS) was 2.7 months and the 
1-year OS probability was $67 \%$. Notably, all patients enrolled in NIVOREN have at least one lesion $>5 \mathrm{~mm}$ to assess for response, and it remains to be seen how these agents may impact patients with lower disease burden. Preliminary results evaluating combination ipilimumab and nivolumab (CheckMate 920; ClinicalTrials.gov identifier: NCT02982954) in patients with mRCC and asymptomatic brain metastases are encouraging, with a preliminary reported PFS of 9 months and a systemic objective response rate of $28.6 \% .^{24}$ Furthermore, preliminary post hoc analyses from JAVELIN Renal 101, the phase III study of axitinib + avelumab versus sunitinib, have shown a similar observed PFS between treatment groups in patients who enrolled with brain metastasis. ${ }^{25}$

Although the evaluation of mandatory trial screening offers a standardized opportunity to limit clinician biases in screening for brain metastases, a major limitation of this dataset remains a selection bias for those patients who considered enrollment in a clinical study and the limited total cohort size for robust comparative analyses. Because this study was performed across several clinical trials, it is important to note that the absence of documented neurologic symptoms is not a substitute for dedicated neurologic evaluation or interview. Furthermore, in addition to other limitations inherent in retrospective analyses, capturing all treatment-related follow-up remains incomplete and comparison between this asymptomatic patient population and those diagnosed with symptomatic disease is challenging. Further work that compares these 2 populations will be integral in reforming clinical decision tools and management guidelines at the population level. For instance, this dataset highlights a higher incidence of brain metastases in previously treated patients, those with metastatic disease at initial presentation, and those with lung involvement. These characteristics may serve as crude metrics to identify high-risk patient populations in whom screening would add value.

In sum, this study highlights that a relevant proportion of patients with mRCC may harbor occult brain metastases, of which most were found at study screening in the treatment-refractory setting. These results provide perspective for treatment guidelines that impact active surveillance or treatment paradigms in patients with advanced disease. Given that this site of diagnosis is often associated with high morbidity, mortality, and urgent multidisciplinary care, these data provide support for broadly applied screening measures, particularly in patients who experience disease progression on first-line therapies, those who initially present with metastatic disease at diagnosis, and those with pulmonary metastases or several sites of extracranial disease. Further efforts that improve on current screening decision trees to identify patients with occult brain disease may allow early intervention and therapeutic action.

\section{Conclusions}

This study highlights that $4 \%$ to $5 \%$ of patients with mRCC may harbor metastatic occult brain disease. Neither incidence nor outcomes were apparently associated with IMDC risk status in this cohort. Screening should be considered for patients with high metastatic burden or those who have experienced disease progression after first-line therapies. Consistent evaluation of risk and identification of algorithmic screening approaches may further characterize high-risk populations.

Submitted June 29, 2020; accepted for publication August 4, 2020. Published online February 12, 2021.

Author contributions: Study concept: Kotecha, Escudier, Motzer, Albiges, Voss. Study design: Kotecha, Flippot, Escudier, Motzer, Albiges, Voss. Data acquisition: Kotecha, Flippot, Nortman, Guida. Quality control of data and algorithms: Kotecha, Flippot, Albiges, Voss. Data analysis and interpretation: Kotecha, Flippot, Escudier, Motzer, Albiges, Voss. Statistical analyses: Kotecha, Flippot, Patil. Manuscript preparation: Kotecha, Flippot, Escudier, Motzer, Albiges, Voss. Critical revision: All authors.

Disclosures: Dr. Escudier has disclosed that he is a scientific advisor for Novartis, Pfizer, Bristol-Myers Squibb, Ipsen, EUSA Pharma, AVEO, and Genentech; receives honoraria from Pfizer, Novartis, Bristol-Myers Squibb, Ipsen, Genentech, EUSA Pharma; and receives consulting fees from BristolMyers Squibb, Pfizer, Genentech, and Ipsen. Dr. Motzer has disclosed that he receives consulting fees from Pfizer, Eisai, Merck, Genentech (Roche), and Novartis. Dr. Albiges has disclosed that he is a scientific advisor for Novartis, Amgen, Bristol-Myers Squibb, Ipsen, Roche, Novartis, Pfizer, Astellas Pharma, and Merck. Dr. Voss has disclosed that he receives grant/research support from Bristol-Myers Squibb, Pfizer, and Genentech; is a scientific advisor for Alexion Pharmaceuticals, Calithera Biosciences, Corvus Pharmaceuticals, Exelixis, Eisai, Natera, Novartis, and Pfizer; receives consulting fees from Eisai and Takeda; and receives honoraria from Novartis. The remaining authors have disclosed that they have not received any financial consideration from any person or organization to support the preparation, analysis, results, or discussion of this article.

Correspondence: Martin H. Voss, MD, Department of Medicine, Memorial Sloan Kettering Cancer Center, 300 East 66th Street, Box 138, New York, NY 10065. Email: vossm@mskcc.org

\section{References}

1. Schouten LJ, Rutten J, Huveneers HA, et al. Incidence of brain metastases in a cohort of patients with carcinoma of the breast, colon, kidney, and lung and melanoma. Cancer 2002;94:2698-2705.

2. Daugherty M, Daugherty E, Jacob J, et al. Renal cell carcinoma and brain metastasis: questioning the dogma of role for cytoreductive nephrectomy. Urol Oncol 2019;37:182.e9-182.e15.

3. Shuch B, La Rochelle JC, Klatte T, et al. Brain metastasis from renal cell carcinoma: presentation, recurrence, and survival. Cancer 2008; 113:1641-1648.

4. Bowman IA, Le T, Christie A, et al. Incidence of brain metastases in metastatic renal cell carcinoma in the era of targeted therapies [abstract]. J Clin Oncol 2016;34(Suppl):Abstract e16103.

5. Hanzly M, Abbotoy D, Creighton T, et al. Early identification of asymptomatic brain metastases from renal cell carcinoma. Clin Exp Metastasis 2015;32:783-788

6. Bianchi $M$, Sun $M$, Jeldres $C$, et al. Distribution of metastatic sites in renal cell carcinoma: a population-based analysis. Ann Oncol 2012;23 973-980. 
7. Motzer RJ, Jonasch E, Agarwal N, et al. NCCN Clinical Practice Guidelines in Oncology: Kidney Cancer, Version 2.2020. Accessed August 12, 2020. To view the most recent version, visit NCCN.org

8. Ljungberg B, Albiges L, Abu-Ghanem Y, et al. European Association of Urology guidelines on renal cell carcinoma: the 2019 update. Eur Urol 2019;75:799-810.

9. Escudier B, Porta C, Schmidinger M, et al. Renal cell carcinoma: ESMO clinical practice guidelines for diagnosis, treatment and follow-up. Ann Oncol 2019;30:706-720.

10. de Velasco G, Bex A, Albiges L, et al. Sequencing and combination of systemic therapy in metastatic renal cell carcinoma. Eur Urol Oncol 2019; 2:505-514.

11. Rini BI, Plimack ER, Stus $V_{\text {, et }}$ el. Pembrolizumab plus axitinib versus sunitinib for advanced renal-cell carcinoma. N Engl J Med 2019;380: 1116-1127.

12. Choueiri TK, Escudier B, Powles T, et al. Cabozantinib versus everolimus in advanced renal-cell carcinoma. N Engl J Med 2015;373:1814-1823.

13. Sun M, De Velasco G, Brastianos PK, et al. The development of brain metastases in patients with renal cell carcinoma: epidemiologic trends, survival, and clinical risk factors using a population-based cohort. Eur Urol Focus 2019:5:474-481.

14. Tsui KH, Shvarts $O$, Smith RB, et al. Renal cell carcinoma: prognostic significance of incidentally detected tumors. J Urol 2000;163:426-430.

15. Choueiri TK, Motzer RJ. Systemic therapy for metastatic renal-cell carcinoma. N Engl J Med 2017;376:354-366.

16. Ferrel EA, Roehrig AT, Kaya EA, et al. Retrospective study of metastatic melanoma and renal cell carcinoma to the brain with multivariate analysis of prognostic pre-treatment clinical factors. Int J Mol Sci 2016;17:400.
17. Motzer RJ, Tannir NM, McDermott DF, et al. Nivolumab plus ipilimumab versus sunitinib in advanced renal-cell carcinoma. N Engl J Med 2018;378: 1277-1290.

18. Motzer RJ, Penkov K, Haanen J, et al. Avelumab plus axitinib versus sunitinib for advanced renal-cell carcinoma. N Engl J Med 2019;380: 1103-1115.

19. Juloori A, Miller JA, Parsai S, et al. Overall survival and response to radiation and targeted therapies among patients with renal cell carcinoma brain metastases [published online January 18, 2019]. J Neurosurg, doi: 10.3171/2018.8.JNS182100

20. Albiges L, Flippot R, Arfi-Rouche J, et al. Brain metastases (BM) from renal cell carcinoma treated with nivolumab: evidence of early brain flare? [abstract]. J Clin Oncol 2017;35(Suppl):Abstract 520.

21. Négrier S, Moriceau G, Attignon $V$, et al. Activity of cabozantinib in radioresistant brain metastases from renal cell carcinoma: two case reports. J Med Case Reports 2018;12:351.

22. Chevreau C, Ravaud A, Escudier B, et al. A phase II trial of sunitinib in patients with renal cell cancer and untreated brain metastases. Clin Genitourin Cancer 2014;12:50-54.

23. Flippot R, Dalban C, Laguerre B, et al. Safety and efficacy of nivolumab in brain metastases from renal cell carcinoma: results of the GETUG-AFU 26 NIVOREN multicenter phase II study. J Clin Oncol 2019;37:2008-2016.

24. Emamekhoo H, Olsen M, Carthon BC, et al. Safety and efficacy of nivolumab plus ipilimumab (NIVO+IPI) in patients with advanced renal cell carcinoma (aRCC) with brain metastases: interim analysis of CheckMate 920 [abstract]. J Clin Oncol 2019;37(Suppl):Abstract 4517.

25. Jonasch E, Hasanov E, Motzer RJ, et al. Evaluation of brain metastasis in JAVELIN Renal 101: efficacy of avelumab + axitinib $(A+A x)$ versus sunitinib (S) [abstract]. J Clin Oncol 2020;38(Suppl):Abstract 687. 